\title{
Open Developmental Science: An Overview and Annotated Reading List
}

\author{
Tamara Kalandadze ${ }^{1}$ and Sara A. Hart ${ }^{2,3}$ \\ ${ }^{1}$ Department of Education, ICT and Learning, Østfold University College, Norway \\ ${ }^{2}$ Department of Psychology, Florida State University \\ ${ }^{3}$ Florida Center for Reading Research, Florida State University
}

\section{Author Note}

Tamara Kalandadze (Dhttps://orcid.org/0000-0003-1061-1131

Sara A. Hart (iD https://orcid.org/0000-0001-9793-0420

We have no conflict of interest to disclose.

This work was supported in part by Eunice Kennedy Shriver National Institute of Child Health \& Human Development Grants HD052120 and HD095193. Views expressed herein are those of the authors and have neither been reviewed nor approved by the granting agencies.

Correspondence concerning this article should be addressed to Tamara Kalandadze, Department of Education, ICT and Learning, Østfold University College, Norway, P.O Box 700, NO-1757 Halden Norway,Email:tamara.kalandadze@ hiof.no

\section{Contribution roles according to the CRediT taxonomy:}

Tamara Kalandadze: Conceptualization; formal analysis; methodology; project administration; visualization; Writing-Original draft preparation

Sara Hart: Conceptualization; formal analysis; methodology; supervision; visualization; Writing-Original draft preparation 


\begin{abstract}
The increasing adoption of open science practices in the last decade has been changing the scientific landscape across fields. However, developmental science has been relatively slow in adopting open science practices. To address this issue, we followed the format of Crüwell et al., (2019) and created summaries and an annotated list of informative and actionable resources discussing ten topics in developmental science: Open science; Reproducibility and replication; Open data, materials and code; Open access; Preregistration; Registered reports; Replication; Incentives; Collaborative developmental science.

This article offers researchers and students in developmental science a starting point for understanding how open science intersects with developmental science. After getting familiarized with this article, the developmental scientist should understand the core tenets of open and reproducible developmental science, and feel motivated to start applying open science practices in their workflow.
\end{abstract}

Keywords: Open Science, Developmental Science, Developmental research, Open Research, Preregistration, Registered reports, Developmental psychology 


\section{Open Developmental Science: An Overview and Annotated Reading List}

Robust and actionable knowledge generated by rigorous and reliable developmental science is essential for accumulating knowledge about the development, health, and wellbeing of humans (Gilmore et al., 2020; SCRD, 2019). However, developmental science shares many of the features identified as problematic in other fields (e.g., Davis-Kean \& Ellis, 2019). Some of these features might be even more expressed in developmental research due to a number of methodological and analytical choices researchers make in the research process (Frank et al., 2017; Silverstein, 2020). These problematic features create barriers to the adoption of best scientific practices, and lead to a lack of cumulativeness in the field. Identifying and addressing the barriers to adopting best scientific practices is vital to improving the value of developmental science and the knowledge it delivers.

Open science (also referred to as open research and open scholarship) has been proposed as one of the solutions or measures to address the problems in (psychological) science and to increase the quality and value of research findings. Open science is a framework of beliefs and principles, as well as practices promoting the openness, reproducibility, replicability, transparency, and rigor of research (Asendorpf et al., 2013; Crüwell et al., 2019; Kathawalla et al., 2020; Munafò et al., 2017; Syed, 2019). The uptake of open science beliefs and practices has been relatively slow and uneven throughout the subfields of psychology (Norris \& O’Connor, 2019; Syed \& Kathawalla, 2022). The unevenness in awareness, acceptance, and implementation of open science practices may result in an asymmetry of rigor and robustness of research across the fields (Syed \& Kathawalla, 2022). Therefore, identifying why some subfields are slow in adopting open science practices, and suggesting ways to improve the current practices is valuable. 
OPEN DEVELOPMENTAL SCIENCE

Developmental science is one of the subfields that has not incorporated open science practices to the same degree as we have seen in, for example, social psychology. This is problematic because the specificities of developmental research (e.g., difficulties to recruit participants leading to underpowered studies; e.g., Peterson, 2016; messy data, strong ethical concerns) make the field particularly vulnerable concerning questionable research practices (QRPs; John et al., 2012) identified in psychology in general (Davis-Kean \& Ellis, 2019; Frank et al., 2017; Silverstein, 2020). This vulnerability of developmental science makes it obvious that developmental science can benefit from embracing open science practices (Gilmore \& Quian, 2021; Silverstein, 2020). The slow adoption of open science practices may place developmental research at risk of falling behind the latest developments in (psychological) research.

The Society for Research in Child Development (SRCD) considers Scientific integrity, Transparency, and Openness, as applied to research, to the teaching of scientific methods, and the translation of science into practice and policy, as core values and strongly endorses them (SRCD, 2019). According to its policy, emphasizing openness and transparency will yield more robust and reliable findings that will serve both future research, as well as stakeholders such as parents, the public, and policymakers (SRCD, 2019). However, an agreement on values and/or goals does not guarantee agreement about what specific practices represent the values most effectively or advance the goals (Gilmore et al., 2020; Nosek et al., 2015). SRCD acknowledges that no single set of practices can apply to the diversity of research conducted within development science (SRCD, 2019). We believe that selecting appropriate research practices for particular projects requires awareness and knowledge of different practices by developmental science researchers, awareness and knowledge that is not necessarily currently available. 
OPEN DEVELOPMENTAL SCIENCE

Lack of information about open science practices has been identified as one of the primary reasons that researchers in psychology are not using the best scientific approaches (Crüwell et al., 2019; Washburn et al., 2018). Sometimes, researchers do find relevant resources but feel overwhelmed by the number of resources and the difficulty to digest their content because some of these resources might be too technical to understand (Crüwell et al., 2019; Parsons et al., 2021). This might result in not knowing "how to start" and lead to a sense of paralysis (Kathawalla et al., 2020) or even thinking that learning about open science practices is time-consuming and not feasible. Considering the time constraints of the researchers, learning about and integrating open science practices can be seen as an extra burden on the top of already busy schedules (FORRT, 2019). Limited awareness of different practices and lack of knowledge on how to implement them, combined with other factors (e.g., current incentives that do not endorse best practices; Bakker, van Dijk, \& Wicherts, 2012; Higginson \& Munafò, 2016; Travers et al., 2016) can be thought of as slowing down the process of adaptation of these practices in psychology, including developmental science.

To address these barriers that prevent developmental scientists from applying open science practices, a shared understanding and awareness of these practices and the values they present is essential. Therefore, accessible overviews introducing open science topics are valuable (e.g. Crüwell et al. 2019; Kathawalla et al., 2020). 
To support researchers starting to implement open science practices, Crüwell et al., (2019) created the guide that outlined the best openly accessible resources related to improved practices in (psychological) science. However, considering the specific features of developmental science, tailoring such a guide specifically for researchers and students in developmental science is needed. Concerns about data sharing, such as privacy violations of research participants, ethics board approvals, and risks of being scooped by other researchers are common among developmental researchers (Gennetian et al., 2020). To address these concerns, and to reduce barriers to understanding these practices, we provide an overview of relevant resources in the format of summaries and annotated reading list of selected open science resources specific to developmental science. Inspired by the format of an annotated reading list used by Crüwell et al., (2019), we have collated and summarized resources discussing open science practices in developmental research including subfields such as infant research, and educational psychology. The project also has its OSF-page (https://osf.io/z24s3/).

We hope that this approachable introduction to open science practices in developmental science will be a welcome resource or entry for researchers at all career stages who are new to open science and that it will help them in navigating discussions of open developmental science. The paper will also be a useful resource for teaching open science to students in developmental psychology, (special) education, educational psychology, and beyond. Importantly, this paper can also inform conversations about open science in general.

\section{Methods of annotated reading list and summary writing}

We have been collating all relevant resources regardless of type (e.g., articles, tutorials) to identify what open science principles or practices have been discussed in the context of developmental science, and to collect them in one place. We started by adding the resources that we were familiar with and then searched the (special issues in) relevant 


\section{OPEN DEVELOPMENTAL SCIENCE}

journals. We also searched in the publicly shared curated reading lists, preprint servers, by searching relevant authors as well as the reference lists of the identified resources, and by asking on Twitter. In line with the focus of this special issue on diversity, we intended to include resources discussing various research methods and open science practices of both "typical" and "atypical" development from the prenatal period through emerging adulthood.

We then grouped our curated reading list into topics. We started with the seven open science topics covered in Crüwell et al., (2019): understanding open science; open access; open data, materials and code; reproducible analyses; preregistration and registered reports; replication research; and teaching open science. We also noticed two other themes emerged from our curated reading list: incentives, and collaborative developmental science.

From this curated reading list, we selected one source per topic which we felt was informative, and was the most general to all of developmental science. For example, when faced with sources that covered a topic for just infant researchers versus all developmental sciences, we selected the latter. However, in the absence of sources discussing the practices in developmental science in general, we were flexible and summarized sources discussing these topics in specific subfields (e.g., infants). Note that although some readings might be from specific subfields, much of it can be generalized to other subfields of (developmental) science.

During this selection process, we made two changes to our original topics. We slightly changed "reproducible analyses" to "reproducibility and replication", according to the source we summarized. Also, rather than a single source covering both "preregistration and registered reports", we had two different sources for these topics and decided to split the topic. The final topics the summarized resources cover are: Open science; Reproducibility and replication; Open data, materials and code; Open access; Preregistration; Registered reports; Replication; Incentives; Collaborative developmental science. 
OPEN DEVELOPMENTAL SCIENCE

After selecting the final source for each topic, we wrote an accessible summary of the essential ideas discussed in each source, with a short introduction and conclusion for each topic. The summaries will allow readers to determine if consulting the source would be useful for them. We also recommend other relevant sources on these topics within each summary.

\section{Summaries}

\section{Open science}

Source: van Dijk, W., Schatschneider, C., \& Hart, S. A. (2021). Open Science in Education Sciences. Journal of Learning Disabilities, 54(2), 139-152. https://doi.org/10.1177/0022219420945267

Open science is a buffet of practices that developmental science researchers can choose from, with the ultimate goal of adopting as many as possible. However, just as the field of developmental science is varied, so are the open science practices. They vary in when they are used, as well as the time, resource, and expertise intensity of using the practices. Our first paper, van Dijk, Schatschneider and Hart (2020) summarizes the most five common open science practices, which are also recognizable as the open science badges (i.e, Center for Open Science): Open Data, Open Analysis, Open Materials, Preregistration, and Open Access. Van Dijk et al. focus their review for educational scientists, but we believe that it applies to almost any developmental sciences researcher.

Van Dijk et al. begin with a review of each of the five open science practices, including useful definitions of each and citations. Following this, the authors then review the history of open science. They remind us that although open science practices became part of the common discourse in educational science (and therefore developmental sciences) because of recent high-impact replication failures in psychological sciences, concerns about the scientific practices of developmental science have been around for decades. For example, research in the 1960s discussed the issues of selective outcome reporting and hypothesizing after results are known (HARKing) (Meehl, 1967). Van Dijk et al. also point out that many 
OPEN DEVELOPMENTAL SCIENCE

open science practices are not new, for example, we have always regularly made our data open via summary statistics.

Van Dijk et al. then review two benefits of open science practices. The first is that open science practices increase transparency in our science, in particular, increasing our ability to produce scientific evidence that is reproducible. For readers who are interested in a longer summary of the importance of open science to increase the credibility, equity, impact, and efficiency of educational, and developmental science, research, we recommend Fleming et al. (2021) for a good review of open science practices from the transparency lens. The second benefit van Dijk et al. review is the ability to use open science practices to develop and answer novel research questions. As an example, the authors describe the usefulness of the Child Language Data Exchange System (CHILDES), an open data repository of child language transcripts.

Van Dijk et al. then review resources and provide a "how to' for each of the five open science practices. The authors include a useful graphic that lays out the key details of how to do each open science practice at different stages of the research process, from project design to after a project is completed. We believe that at this stage of the open science movement, many readers may know something about open science practices, but are less knowledgeable of how to do it in their own work. This part of the van Dijk et al. paper will be especially useful for readers, and we also guide readers to Cook et al. (2021), who also have a useful figure with a brief overview of how and when to do different open science practices.

Finally, van Dijk et al. summarize their recommendations for the field. First, they remind all researchers that they can adopt open science practices. They suggest sharing data and materials from projects which have been completed as a good first step. They also encourage researchers to promote open science practices and to encourage colleagues during 
collaborative projects or hallway conversations. Van Dijk et al. also suggest that researchers can support open science practices during grant and paper reviewing.

We highlight sources that are specific to certain open science practices in the remainder of our paper, but having a general overview of all of the practices is useful for readers, especially those new to the area or teaching students new to the area. The value of open science can be described as increasing the rigor and transparency of a field, but also it can be described as good for the researcher and for other researchers in the field (e.g., Markowetz, 2015).

\section{Reproducibility and replication}

Source: Davis-Kean, P. E., \& Ellis, A. (2019). An overview of issues in infant and developmental research for the creation of robust and replicable science. Infant behavior \& development, 57, 101339. https://doi.org/10.1016/j.infbeh.2019.101339

Although open science practices are not new, the genesis for the growing movement of using open science practices in psychology, and subsequently developmental science, is attributed to the "replication crisis" (e.g., Pashler \& Wagenmakers, 2012; Simmons et al., 2011). Stemming from the replication crisis, there was a movement to make psychology more rigorous, and the findings reproducible (i.e., recreate findings in the same dataset) and replicable (i.e., recreate findings in a different dataset). That movement was heavily tied to using open science practices. Before we move into different open science practices, we review here a piece that summarizes the questionable research practices common in developmental science (Davis-Kean \& Ellis, 2019).

The authors begin their piece with a history of when the replication crisis became apparent in psychology, including citing work that showed how many developmental science papers have $p$-values that are incorrectly reported (i.e., Bakker \& Wicherts, 2011). This then sets up their paper, which reviews questionable research practices in developmental science in 
three categories: statistical power and sample size, protocol flexibility, and analysis flexibility (Davis-Kean \& Ellis, 2019).

Davis-Kean and Ellis. begin the statistical power and sample size section with a review of how difficult it is to determine appropriate expected effect sizes for power analysis in developmental science. Small sample sizes in the existing literature mean that published effect sizes are artificially inflated, and in general, effect sizes in developmental science are small meaning rules of thumb, such as Cohen's effect size magnitudes, don't apply well. The authors suggest some strategies to support developmental scientists in their estimation of effect sizes for power analyses. First, they recommend drawing on the literature for the range of effect sizes but suggest that researchers focus on larger sample studies when doing so. The authors then note that often there is not literature to draw from, and in situations like this, they recommend using an effect size of .10 for power analysis, to be conservative. The authors work out an example of what sample size would be required to test the difference between two groups with a low effect size, and the result is sample sizes that pretty much no one lab could do in developmental science (1250 participants per group, for 2500 total participants). This leads to the suggestion that developmental scientists should collaborate across labs or otherwise pool data using existing data repositories.

The second section of the Davis-Kean and Ellis paper focuses on protocol flexibility. This section reviews what's been called "researcher degrees of freedom" (Wicherts et al., 2016), the researcher decisions made at each stage of the research process that are not noted or often even recognized as important. The authors review how easy it is for developmental scientists to go "off protocol" when testing children, and that often these changes to the protocol are not written down. If the changes to the protocol are done systematically, they can lead to bias in the results. The authors suggest that researchers have a method to note any 
changes to the protocol so that they can be reviewed (e.g., an online lab notebook), or to even videotape all testing sessions so that protocol deviations can be reviewed.

The third section focuses on analysis flexibility. Davis-Kean and Ellis. review how the difference between confirmatory and exploratory analyses is becoming less distinct. They also review various ways researchers are $p$-hacking, manipulating data to find a statistically significant finding, as well as HARKing, hypothesizing after the results are known. The authors summarize what they think is the most common instance of analysis flexibility in developmental science, which is multiple analyses conducted without hypotheses to support them, such as looking at large correlation matrices and picking out the statistically significant relations to write up. Davis-Kean and Ellis. also review how this can happen during the review process, that reviewers or editors can make authors do extra statistical tests or change variables, a difficult problem to overcome. The authors suggest that researchers can be clear in their manuscript what analyses are exploratory versus confirmatory in cases like this.

The authors then move on to their suggestions for best practices for future developmental science research. They suggest that preregistrations and Registered Reports are the best way to move forward towards a rigorous and reproducible developmental science. We agree with the authors.

\section{Data and material sharing}

Source: Gilmore, R. O., \& Qian, Y. (2021). An open developmental science will be more rigorous, robust, and impactful. Infant and Child Development, e2254. https://doi.org/10.1002/icd.2254

Data sharing, a key open science practice, is the process by which research data is made available for other researchers to examine or use. It may be argued that data sharing is the practice that has the greatest top-down support, in that many major grant funders and journals are now requiring data sharing for grantees and authors. However, in practice data sharing remains a time-intensive practice which requires some specialized skills, resulting in less 
uptake by researchers than other open science practices (e.g., Makel et al., 2019). Often linked with data sharing is material sharing, including code sharing, which involves researchers sharing study materials in an online repository. We could only find one paper that discusses data and material sharing from a developmental perspective, Gilmore and Qian (2021), which we will review here.

Gilmore and Qian. begin by stating they intend to show why open sharing of data and research procedures and methods will strengthen developmental science. They note that developmental scientists should embrace values of transparency and openness so that we might best understand behavior rigorously and transparently.

The authors then have two sections, one for data sharing, and one for sharing of methods and materials. To start the data sharing section, Gilmore and Qian. note that in the history of science, sharing datasets was common. Although it is not currently common in developmental science, the authors believe that it should be again. Specifically, they believe that the data underlying every published paper should be openly shared so that other analysts can check the rigor of published work. The authors then go on to answer the What, When, Where, and How of data sharing. For What, they define what data should be shared, which they state should be at the minimum all published data. For When, they suggest that data should be shared as early as possible, but practically shared when a publication is submitted. For Where, data should be shared as supplemental material, in a journal specializing in publishing data, or a data repository. For How, data should be shared using FAIR (e.g., Wilkinson et al., 2016) and well-documented to support data reuse.

The authors then go on to describe "active curation", when data are uploaded into a data repository as soon as they are collected. They give an example using their own data repository, Databrary, which uses a special R package to allow access to data stored in the data repository, allowing for reproducible analytical workflows. The authors conclude the 


\section{OPEN DEVELOPMENTAL SCIENCE}

data sharing section with two concerns. First, "post hoc curation" is difficult. Gilmore and Qian. strongly recommend that researchers plan for data sharing at the start of their project to spread the efforts required to share data across the lifecycle of the project. The authors also give some brief recommendations surrounding data management guides. For readers who are interested in more detail on how to share their data, we recommend other readings, including Logan et al. (2021) and Meyer (2018). Second, developmental scientists often collect sensitive data, and ethical concerns must be met. Gilmore and Qian. give some references for practical guidance, as well as a link to templates they've created (see also

https://ldbase.org/resources). They also describe how developmental scientists should think about consent when considering data sharing.

The second section of the Gilmore and Qian. paper focuses on sharing of methods and materials. They begin by noting that it's not easy to reproduce most publications, which is a concern for the field. They encourage researchers to share research protocols openly on the Internet using free and open-source software tools, like Markdown or GitHub. They also encourage sharing of data analysis pipelines, including code and data documentation, for future reuse. This section includes some links and suggestions for how to share materials, but it is brief. For readers interested in more details, we could not find a stand-alone paper focused on materials sharing for developmental scientists, but recommend the appropriate section within Cook et al. (2021) for some more how-to's.

Gilmore and Qian. is a brief introduction to data and material sharing. We have highlighted other readings for those who want more in-depth information about either. In addition, we recognize that all the cited work thus far is biased towards quantitative research. For those who would like more information about data and material sharing for qualitative research, we recommend Tsai et al., (2016), and Mannheimer et al., (2019). 


\section{Open Access}

Source: Roehrig, A. D., Soper, D., Cox, B. E., \& Colvin, G. P. (2018). Changing the default to support open access to education research. Educational Researcher, 47(7), 465-473

Open access is an open science practice rooted in the idea that scientific findings should be easily and freely available to everyone online, not just those who have access to paywalled journals. We selected a paper to review that is written for an educational sciences audience but applies to all developmental sciences (Roehrig et al., 2018). The paper's authors are a unique combination of educational scientists and librarians, and it summarizes the concepts, rationale, and areas of resistance around open access.

Roehrig et al. start with a description of what Open Access (OA) is. They start with some general OA terms, and also note that only approximately a quarter of all recently published scholarly products are available OA, meaning the vast majority of our science is behind a paywall where only certain people can access it. The authors then define "Gold OA" publishing (i.e., making a scholarly product freely available at publication by the original publisher), and "Green OA" (i.e., authors personally posting their scholarly product into a repository or other website). For Gold OA, they further differentiate "Diamond OA", which are publishers that allow for free publication, from commercial OA publishers, which charge a fee for publication to generate revenue, from "Hybrid OA", which are traditional publishers who allow authors to pay fees to make their paper OA. For Green OA, the authors further discuss some issues around self-archiving, including publisher policies, and slow scientific community uptake.

In the next section of their paper, Roehrig et al. go on to describe why OA has been growing, although growing slowly. They describe various federal mandates for OA policies, as well as institutional policies to support OA adoption. The authors note that many education research journals do not have OA publishing availability (for empirical work on OA policies in special education journals, see Fleming \& Cook, 2021), and Green OA has not been widely 
taken up by the field. The authors spend some time examining why OA has been slow to catch on in educational sciences, suggesting that it's due to the lower journal prestige of newer Gold OA journals coupled with the relative lack of OA journals. We note that since this paper has been published, an education-oriented Green OA data repository, EdArXiv has been started, and is showing greater recent use. The authors believe that OA is not widely supported or encouraged by research-intensive universities, meaning there is not a reward structure in place to change the culture around OA publishing. In addition, the authors describe why they believe that traditional publishers are making their publication contracts purposely restrictive against Green OA.

Roehrig et al. then give their recommendations for how the default negative thoughts towards OA can be changed. First, the authors point to the success other countries outside of the US have had with top-down mandates from funders and institutions, and they recommend similar mandates in the US, with accompanying compliance mechanisms. The authors note that OA mandates in other countries have led to more permissive copyright policies from publishers.

The authors note that they continue to see reluctance by researchers to use OA publishing options, and they finish their article by stating common apathy and fears they've heard from researchers about OA (e.g., "I don't have time"), and their rationale for how to overcome these. The authors believe that moral arguments will not be enough to sway reluctant authors and that practical arguments are needed. The authors give an anecdote of showing a colleague the large engagement they've had with an OA paper, which could be coupled with the meta-science research showing OA papers have more downloads than paywalled articles (e.g., Davis et al., 2008). The authors also call on senior faculty to support OA publishing, to challenge the current system of blindly signing copyright forms without questioning them, and supporting their pre-tenure colleague's support of OA. Roehrig et al. 
also call on Editors of journals to look at the copyright forms of their journals and to apply pressure to journals to change their policies if they are restrictive to OA publishing. The authors include a useful table on things stakeholders can do to change from the current default of no OA to allowing OA publishing. The authors conclude with a call to our community to support OA publishing as a service to the academy. We second this call and point out that Green OA is easily available to all readers, such as posting the final accepted manuscript document (before copyediting/page proofs) to a repository such as PsyArXiv or EdArXiv. As developmental scientists, we are interested in our science helping children, families, and communities, and if our science is behind a paywall, we cannot reach our stakeholders.

\section{Preregistration}

Source: Havron, N., Bergmann, C., \& Tsuji, S. (2020). Preregistration in infant research-A primer. Infancy, 25(5), 734-754

Preregistration, an open science practice referring to specifying a research plan before conducting a study, is increasingly used in research. Preregistration has been suggested as one way of preventing researchers from unintentionally engaging in questionable research practices (Nosek et al., 2018), and reduces researcher degrees of freedom, meaning that researchers can choose from many different possible choices for analysis, exclusion criteria, and the like.

Our next source, Havron et al., (2020) presents an in-depth discussion of the issues that might make preregistration of infant research particularly challenging, but discusses also ways to contend these issues. Havron et al. believe that a priori examination of the planned study increases the credibility of single studies and adds value to the field.

Havron et al. describe the problems with transparency and reproducibility in infant studies and explain how preregistration offers a particular advantage to infant researchers. By carefully deciding on the design, analyses, etc., researchers are less likely to conduct a study and discover errors after completion of data collection. 
For an individual researcher, preregistration can have several practical advantages such as avoiding duplicating work and contributing to more efficient workflows. Sharing analysis scripts along with preregistration makes it easier to understand the purpose of these scripts, but they also become reusable for others. Detailed documentation of the research process in preregistration can also prevent mistakes.

Among the challenges with preregistration, Havron et al. highlight the importance of describing deviations from a preregistration. This is important because if undisclosed, deviations do not increase transparency, and are even considered questionable scientific practice. Another issue with undisclosed deviations is that authors might falsely profit from the credibility that preregistration is associated with, and as such, they can potentially undermine the credibility of preregistration. Another, but related problem is imprecisely formulated preregistrations, which increase researcher degrees of freedom. The absence of a standard for preregistration makes it difficult to know what the appropriate level of specificity in preregistrations is.

Havron et al. remind us that preregistration does not automatically improve a flawed research question or planned methodology. The value of preregistration depends on whether the preregistration plans are followed when conducting the study, and whether any deviations from the preregistration are reported.

Havron et al. then briefly discusses registered reports (RRs), that is submitting a preregistration in the form of the Introduction and Methods section of a journal article which undergoes several rounds of peer review. Among the advantages of RRs is that acceptance means almost guaranteed publication, which is not based on whether outcomes are significant. RRs, thus, prevent QRPs. Another advantage with RRs is that, as a result of the expert peer review, the method is likely to be improved. However, RRs also have a disadvantage that is the time needed for peer review which can make RRs unrealistic for researchers on a 
temporary contract (see, however, Hobson, 2019). Havron et al. note that, like any other format, RRs are not a cure-all solution as expert review is never a guarantee for a sound research design. Despite this, they recommend infant researchers to consider RRs when feasible because improvements made in the peer-review process are beneficial to infant researchers given the resource-intensive nature of their research.

Then Havron et al. mention the current platforms that allow preregistering online such as Open Science Framework and GitHub (we also refer readers to a list of preregistration templates which make it easier to get started https://osf.io/zab38/wiki/home/).

Finally, Havron et al. write about how researchers can update their preregistrations, in particular, it's possible to upload an amendment to a preregistration before knowing the results. Doing so is by no means a questionable research practice. We think that this is an important point because without knowing about the possibility to submit an amendment to the preregistering detailed plan or to deviate from the preregistration, it may seem daunting and binding to even start (DeHaven, 2017). Luckily, a preregistration is a document of prespecified research plans before seeing the results, and it is not a prison sentence (DeHaven, 2017). The key both in preregistration and in reporting the study, is to be transparent and honest. Although Havron et al. discuss preregistration (and registered reports) specifically for infant research, many of the issues/problems and suggested solutions can be relevant for other sub-fields. For those who would like more information about preregistrations, we recommend to consult Nosek et al. (2018).

\section{Registered reports}

Source: Reich, J., Gehlbach, H., \& Albers, C. J. (2020). "Like upgrading from a typewriter to a computer": Registered reports in education research. AERA Open, 6(2), 1-6. https://doi.org/10.1177/2332858420917640

Registered Reports (RRs) address issues that threaten the integrity of the science. Although one of our summarized sources, Havron et al., (2020) also touched on RRs, it was brief. 
Therefore, we decided to summarize one source on RRs as applied to education research (Reich et al., 2020). Yet, Reich et al. is relevant for other subfields within developmental science and beyond.

RRs are a new format of journal article where authors first submit the manuscript that includes an introduction, background and context, and methods (including prespecified analyses) sections of an article as Phase I. Peer reviewers evaluate the manuscript without knowing what the results will be. Editors can then grant the article "in-principle acceptance," request revisions, or suggest modifications of the study design. An in-principle acceptance means that if the authors adhere to the plan as described in Phase I, then the article will be accepted for publication regardless of the direction of results.

Two important benefits are associated with RRs. First, authors benefit from the expertise provided through the peer review early in the research process, while it is still possible to make methodological changes. Second, in-principle acceptance before results are known prevents QRPs, such as publishing only "significant” results. Over time, adopting RRs should reduce the proportion of false positives in the literature.

Our source, Reich et al. is an introduction to a special issue that includes seven of the first RRs in education research. These articles address diverse topics and uses a variety of methodologies such as lab studies, field experiments, and secondary data analysis. After situating these RRs in the context of this special issue, Reich et al. present author and editor perspectives on RRs. They explain that the editors and authors involved in this Special Topic were enthusiastic about the design, writing, and review process. Both authors and editors agreed that the peer review process was a friendlier, more productive set of exchanges than it typically is in education research publishing. From the editors' perspective, reviewers appeared less critical, and the criticisms were more constructive and generative. Several authors argued that the process of designing a study for a registered report was both easier and 
at the same time more rigorous. Importantly, several authors expressed that the process either took no extra time or saved time compared with traditional publishing. Three studies have graduate students as first authors and authors express the belief that the upcoming generation of education researchers will be champions of new approaches to open science. We agree with this because introducing open science practices early has been shown to benefit both the science and the students (Chopik et al., 2018; Frank \& Saxe, 2012; Stojmenovska et al., 2019; Strand \& Brown, 2018).

Reich et al. continue with discussing challenges and tensions for future Editors of registered reports. Many authors noted, and the editors say they agree, that not all studies are appropriate for a registered report. RRs may be more appropriate for confirmatory research.

Furthermore, all the studies published in the special issue used quantitative methodologies, and the benefits of registered reports for qualitative or design-based work are less clear.

Finally, Reich et al. encourage education researchers to be active in advancing open science in their research through for example writing a proposal to their favorite journal and proposing a special issue on RRs, or planning a registered study and asking their favorite journal to review it as a RR. Finally, authors are encouraged to submit their next article to AERA Open. We second this suggestion and point readers to the list of journals that accept RRs, which is growing every day (e.g., https://www.cos.io/initiatives/registered-reports). We also point readers to a new meta-journal called PCI Registered Reports (https://rr.peercommunityin.org/), which manages the peer review of RRs across fields, and does so quickly. After the peer review is completed and the paper is accepted as a Stage 1 manuscript, authors are welcomed to complete and "publish" their study to the PCI website. Interestingly, some journals collaborate with PCI and pick up these Stage 1 manuscripts, 
OPEN DEVELOPMENTAL SCIENCE

publishing the final Stage 2 manuscript in their traditional journal (e.g., Infant and Child Development). Advances such as these will bring RRs into the mainstream.

\section{Replication}

Source: Plucker, J. A., \& Makel, M. C. (2021). Replication is important for educational psychology: Recent developments and key issues. Educational Psychologist, 56(2), 90-100. doi:10.1080/00461520.2021.1895796

Replication, which is the repetition of previous research to confirm or disconfirm the previous results, is considered as a de facto reliability check on previous research, and as such, a necessary cornerstone of scientific endeavors. Our next source discusses the value and relevance of replication in educational psychology (Plucker \& Makel, 2021).

Replications occur in two forms: direct and conceptual replications. In direct replications, researchers follow the methods of the original study as closely as possible to arrive at similar results. In contrast, researchers doing conceptual replications purposefully alter factors such as demographics, or study context, aiming to examine the theoretical soundness of a particular finding or set of findings. Unfortunately, replications are still uncommon in education, psychology, and educational psychology.

Plucker \& Makel. summarize researchers' current understandings of replications in the following four areas: philosophy of replication, methodology of replication, professional implications of replication, and utility of replication.

Regarding the philosophy of education, although epistemological questions remain, there has been made a non-trivial, philosophical advance regarding the understanding of the value of replications. Plucker \& Makel. argue that replication has well-defined, epistemological purposes and that there is a need for increased use of replications in educational psychology. The authors discuss the value of both direct and conceptual replications and call for explicit statements of intent in the papers regarding the type of replications that are conducted. This subsection ends with disputing the existing view that due 
to the variability in students and contexts, findings in educational psychology research cannot be generalized. Plucker \& Makel. note that numerous findings have indeed been replicated and generalized across contexts.

Under the methodology of replication, the paper explains the similarities (i.e., they both address research quality) and differences between meta-analyses and replications (i.e., they both address research quality in different ways, and solve different problems). In particular, meta-analyses help solve the problem of heterogeneous results, whereas replications help assess and address experimental bias. The paper continues arguing that replication is relevant to all forms of research, including qualitative research.

Under the professional implications of replication Plucker \& Makel. argue that replications are good for one's career. They remind us that more emphasis on replications in educational psychology will help make them more of a key aspect of the educational psychology enterprise. They round out this part by explaining that educational psychology is starting to value and support replication, but the process is slow and uneven.

Regarding the Utility of replication, Plucker \& Makel. remind us that when discussing replications in a field, it is important to discuss whether the most important studies in that field have been replicated, rather than whether a field has replicated a specific percent of studies.

Increased use of replications is feasible and there is no way to create an ideal education system that is informed by research evidence without greater use of replications. Plucker \& Makel. believe that by working together and by using open science practices, educational psychologists will create positive outcomes for both research and practice. Collaboration is the key because it helps to understand existing work better, conduct future research more efficiently and effectively, and provide greater value to practitioners and policymakers. We cannot agree with the authors more. 


\section{Changing Institutional incentives to foster sound scientific practices}

Source: Lundwall R. A. (2019). Changing institutional incentives to foster sound scientific practices: One department. Infant behavior \& development, 55, 69-76. https://doi.org/10.1016/j.infbeh.2019.03.006

One of the main barriers to the adoption of open science practices is the lack of institutional or structural incentives that endorse open science. Most universities provide no incentives to adopt best research practices such as replications, rather they tend to favor novel research findings. It is difficult for researchers, especially pretenure or early career researchers, to practice open science practices when their institutions do not consider for example replication as innovative enough.

A lack of institutional incentives to practice open science may be especially problematic for infant researchers who face unique challenges, such as greater developmental variability across younger age groups and difficulty to recruit participants. Our next source, Lundwall (2021) reports the changes made in incentives to support open science and replication work at their department. Lundwall. reports on specific rationales for restructuring incentives, explains how their new system works and impacts developmental researchers, and provides suggestions for those interested in changing their institutional incentives.

According to Lundwall. these changes were initiated to support sound scientific practices. At their department, few faculty were applying these practices. Ludwell. stresses that this was because researchers felt they needed to focus on institutional expectations for innovation, not because they lacked interest. To remedy this, incentive changes were initiated to support researchers in their efforts. Lundwall. provides a table (Table 3) with examples of activities that increased quality ratings. They believe that providing such a list to faculty early in their careers and prior to annual reviews increases the likelihood that they will be implemented. One advantage of these changed incentives is that they are transparent and from 
the beginning of their employment, a faculty member can know what they should be doing and how they will be evaluated.

Lundwall. explains how the creation of this new 'quality' metric provides a formal method for making a case that a particular study is worth more than might be suggested by simply counting publications. Developmental researchers can now provide evidence that their research activities should count as a technique to improve developmental science, while earlier they needed to remind colleagues evaluating a dossier that developmental studies tend to be costlier and take longer. Although not listed in Table 3, Lundwall. explains that largescale collaborative studies should be supported because they provide more confidence in developmental science by both increasing sample sizes and embracing open science. Developmental science is fundamentally an individual differences research, requiring larger sample sizes. By collaborating with others, researchers may realize the potential of developmental psychology to address problems of development.

These changes allow Lundwall. to be more explicit about methods used and statistical decisions made, and to join large collaboration studies that attempt to replicate key findings in the field. Lundwall provides suggestions for initiating similar changes in other departments and institutions. Table 4 in the article includes a summary of these suggestions (see p.74 in the article).

Lundwall. suggests that, before meeting to discuss a specific plan to change incentives, faculty members should read the literature on open science, replication, reproduction, and similar scientific practices. Also, a seminar on these topics may be especially helpful for busy researchers. Less formal discussions are also valuable. Lundwall. reminds us that in all departments there will be concerns about changes in incentives because they will have various impacts on faculty in different areas. Listening to faculty concerns and providing a transition period and/or flexibility will likely get more people on board. 
Existing incentive structures often put open science and researcher survival in conflict because researchers are expected to provide novel findings. Therefore, institutions should accommodate and incentivize those who want to do replications, given their importance for science. Lundwall. expresses hope that some may follow their lead and begin their process of finding balance between novelty and replication in their work. We agree with Lundwall. in that incentives are important if we want to succeed in adopting open science practices.

\section{Collaborative Developmental Science}

Source: Byers-Heinlein, K., Bergmann, C., \& Savalei, V. (2021, April 8). Six solutions for more reliable infant research. https://doi.org/10.31234/osf.io/u37fy

Science is a highly collaborative endeavor, and working collaboratively has many benefits. Our last source, Byers-Heinlein et al., (2020) shares "lessons emerged" from the first project of ManyBabies - ManyBabies 1 - in which 69 labs from 16 countries tested 2845 infants, of which 2329 were included in the final analysis. Byers-Heinlein et al. also includes personal narratives of the collaborators.

ManyBabies, a large-scale collaboration on key theoretical questions in developmental science, aims to address the problem of small samples by developing and modelling open science practices in infant research such as preregistration, open materials, and open data. Further, ManyBabies aims to increase growth and diversity both within infancy research and in psychological science in general.

Byers-Heinlein et al. describe some of the discussions the contributors had in the process, the solutions they have found, and the challenges that remain. ManyBabies is an example of a collaborative community of infancy researchers committed to open science best practices, operating within a large-scale collaborative research framework or model. This collaborative framework prioritizes collaboration in all aspects of the project.

Byers-Heinlein et al. outline many of the issues, insights, and processes that have emerged over the first few years of collaboration. By providing a behind-the-scenes view of 
the steps and challenges they met in the process, the authors hope that other teams of researchers, in infancy and other fields, will embark on a similar journey, and, not least, that they can learn from their mistakes and benefit from their successes. The insights the authors gained can be applied to future collaborations in psychology at both small and large scales. The authors hope to create greater awareness and uptake of open science practices.

Byers-Heinlein et al. emphasize the role of leadership in collaborative projects because the leadership team plays a crucial role in ensuring that diverse views are heard, steering discussions toward a productive resolution, and pushing the project forward by assigning tasks, setting deadlines, and so on.

Byers-Heinlein et al. describe the strengths and weaknesses of different communication approaches, such as video conference presentations and meetings, and a Slack group. Byers-Heinlein et al. used a model that has been described as a Contributorship rather than an Authorship model (see https://psyarxiv.com/dt6e8/, Holcombe, 2019). However, they did this towards the end of the project which appeared not to be optimal and it might be better to complete while projects are ongoing.

Byers-Heinlein et al. describe what this project achieved proudly, but at the same time, they describe the ongoing challenges they face such as funding and technical infrastructure for large-scale collaborations. Byers-Heinlein et al. also explain the benefits of contributing to open science collaborations, which are often framed in terms of risks to researchers, particularly early-career researchers. One important benefit of ManyBabies is in providing a model of open science in action, implementing research practices such as pre-registration that buffer against the effects of publication bias. In ManyBabies, data are published regardless of the outcome of the data analysis (assuming sound implementation of the experiment). The rich dataset generated by ManyBabies projects allows for secondary analyses. 
Participating in a large-scale collaboration provides researchers with direct access to a community of researchers motivated to develop and share new techniques and best practices in the field. This is particularly important for early career researchers and trainees who may not have as many opportunities to expand their research networks.

ManyBabies continues to grow, despite the challenges the team experienced during ManyBabies 1. The guidelines and best practices that were developed will facilitate success for current and future collaborative projects. They continue to develop networks and are working to ensure that core values of equity, diversity, and inclusion. Byers-Heinlein et al. encourage any interested graduate student or postdoctoral researchers to raise the possibility of participating in ManyBabies with their advisor, and they encourage every principal investigator to find out if somebody in their lab is interested in contributing. The team hopes to collaborate with and learn from a global network of labs from six continents representing the diversity of human experience. They hope that ManyBabies will help serve as a model of how to create collaborative projects to solve the hardest methodological and theoretical problems in the field.

\section{Conclusions and the path forward}

Uptake of open science principles and practices has been uneven across fields. Among the barriers that prevent uptake of open science practices are lack of awareness of, and knowledge about, open science practices. To contribute to reducing these barriers, in this paper we collated and summarized resources describing various open science practices applied in different areas of developmental science. 
OPEN DEVELOPMENTAL SCIENCE

Not only we were inspired by Crüwell et al., (2019), our guide builds on their article, and we recommend readers consult that paper to gain a better understanding of the topics they cover from a general psychology perspective. Other resources we recommend readers get familiarized with are Kattewala et al., (2021), a guide to open science practices for graduate students and their advisors, as well as Kowalczyk et al., (2020), an excellent source targeted towards senior researchers.

Our summarized resources cover the following topics: Open science; Reproducibility and replication; Open data, materials and code; Open access; Preregistration; Registered reports; Replication; Incentives; Collaborative developmental science. Our reading list demonstrates that some practices are discussed more than others in developmental science. For example, we could identify only one white paper (Kim et al., 2020) about teaching open science that also mention developmental scientists. Therefore, we direct readers interested in reading about teaching open science to the excellent initiative-FORRT- Framework for open and reproducible research training (https://forrt.org/). Among several subprojects, the FORRT project includes an excellent collection of open and reproducible science literature, some of which are summarized in an accessible manner.

We want to direct readers' attention to grassroots initiatives in open science such as ReproducibiliTea clubs (https://reproducibilitea.org/) and RIOT Science Club (http://riotscience.co.uk/). One of the authors of this paper coordinates one of the cites of the RIOT Science Club. One of the authors of this paper co-hosts a podcast focused on the methods and metascience of developmental science (Within and Between), which often covers open science topics. In addition, there is a considerable amount of information about open science on the Internet, as well as discussions that occur frequently on social media, especially on Twitter. We suggest searching online for any open science topic to easily find videos of talks as well as open teaching materials, which will be very helpful to readers. 
We also noticed that although we found several resources on special education and learning disabilities, only one source on our reading list was specifically about neurodevelopmental conditions (Farran and Scerif, 2021). There is a clear need for discussions on how to apply open science practices to fields focused on neurodevelopmental conditions.

What was obvious after reviewing the identified resources was that collaboration is considered as vital for researchers wanting to apply open science practices. Indeed, several barriers to the adoption of these practices can be solved through collaborations. No one researcher can be an expert in all the skills needed to do all the open science practices while keeping up with their field and collecting all the data needed for replicable work.

Collaborative science allows the sharing of expertise, time, and resources, making open science more feasible (e.g., Patton Terry et al., 2021).

While we were finishing this paper, we found resources on other important topics such as publishing standards and diversity of experimental samples and researchers (see the supplementary file). We could not summarize these papers, but want to highlight that they are important topics and we encourage readers to read them. In addition, we would like to make it clear that while we focused more on open science as a set of practices, open science is also a set of principles and we recommend readers to get familiar with the historical background, and the fundamentals of open science (e.g., https://osf.io/kgnva/wiki/Open\%20Science\%20Literature/).

To researchers writing about and teaching about open science practices, we wish to recommend using positive approaches to encouraging open science practices (e.g., rather than focusing on those who do not as bad scientists in some way). Open science is not all or none and we cannot expect all researchers to adopt all open science practices in the same projects (see also Syed, 2019). Thinking of open science as a buffet (thanks to Christina Bergmann for 
this metaphor, e.g., https://twitter.com/chbergma/status/1104338904646385665?s=20) means that researchers can pick and choose what researchers can do at that time.

We also note that not all researchers believe open science practices will be good for all, which is a discussion we must have as a field (e.g., Kessler et al., 2021). We recommend readers get familiar with the relevant literature, including the following: Guest (2017), Whitaker \& Guest (2020), Pownall et al. (2021), and Kessler et al., (2021).

Finally, to make it clear, this article does not report a comprehensive literature review based on a systematic literature search. Therefore, some relevant references might be missing, but we have tried to create a comprehensive list as possible and hope researchers and students at all career stages can consult this article to read about open science practices written in an accessible manner. Generally, we agree with Syed (2019) that staying up-to-date on conversations and literature on open science is important because it is about the recognition that we can always improve our work. 


\section{REFERENCES}

Aspendorpf, J. B., Conner, M., De Fruyt, F., De Houwer, J., Denissen, J. J., Fiedler, K., Fiedler, S., Funder, D. C., Kliegl, R., Nosek, B. A., Perugini, M., Roberts, B. W., Schmitt, M., van Aken, M. A. G., Weber, H., \& Wicherts, J. M. (2013). Recommendations for increasing replicability in psychology. European Journal of Personality, 27(2), 108-119. doi:10.1002/per.1919

Bakker, M., \& Wicherts, J. M. (2011). The (mis)reporting of statistical results in psychology journals. Behavior Research Methods, 43(3), 666678. https://doi.org/10.3758/s 13428-011-0089-5

Byers-Heinlein, K., Bergmann, C., \& Savalei, V. (2021, April 8). Six solutions for more reliable infant research. https://doi.org/10.31234/osf.io/u37fy

Chopik, W. J., Bremner, R. H., Defever, A. M., \& Keller, V. N. (2018). How (and whether) to teach undergraduates about the replication crisis in psychological science. Teaching of Psychology, 45(2), 158-163. https://doi.org/10.1177/0098628318762900

Cook, B. G., Fleming, J. I., Hart, S. A., Lane, K. L., Therrien, W., van Dijk, W., \& Wilson, S. E. (2021, March 9). A How-To Guide for Open-Science Practices in Special Education Research. https://doi.org/10.35542/osf.io/zmebaA

Crüwell, S., van Doorn, J., Etz, A., Makel, M. C., Moshontz, H., Niebaum, J. C., ... Schulte-Mecklenbeck, M. (2018, November 16). 7 Easy Steps to Open Science: An Annotated Reading List. https://doi.org/10.31234/osf.io/cfzyx 
OPEN DEVELOPMENTAL SCIENCE

Davis, P. M., Lewenstein, B. V., Simon, D. H., Booth, J. G., \& Connolly, M. J. (2008). Open access publishing, article downloads, and citations: randomised controlled trial. BMJ (Clinical research ed.), 337, a568. https://doi.org/10.1136/bmj.a568

Davis-Kean, P. E., \& Ellis, A. (2019). An overview of issues in infant and developmental research for the creation of robust and replicable science. Infant behavior \& development, 57, 101339. https://doi.org/10.1016/j.infbeh.2019.101339

DeHaven, A. (2017). Preregistration: A Plan, Not a Prison. Retrieved from https://cos.io/blog/preregistration-plan-not-prison/

Farran, E. K., \& Scerif, G. (2021, May 17). Neurodevelopmental conditions, neuroconstuctivism, and reproducible research; challenges and future directions. https://doi.org/10.31234/osf.io/agwkb

Fleming, J. I., \& Cook, B. G. (2021). Open Access in Special Education: A Review of Journal and Publisher Policies. Remedial and Special Education. https://doi.org/10.1177/0741932521996461

Fleming, J.I., Wilson, S.E., Hart, S.A., Therrien, W.J., \& Cook, B.G. (2021). Open accessibility in education research: Enhancing the credibility, equity, impact, and efficiency of research, Educational Psychologist, 56:2, 110-121. doi:10.1080/00461520.2021.1897593

FORRT (2019, December 13). Introducing a Framework for Open and Reproducible Research Training (FORRT). https://doi.org/10.31219/osf.io/bnh7p

Frank, M. \& Saxe, R. (2012). Teaching replication. Perspectives on Psychological Science, 7(6), 600-604. https://doi.org/10.1177/1745691612460686 
OPEN DEVELOPMENTAL SCIENCE

Frank, M. C., Bergelson, E., Bergmann, C., Cristia, A., Floccia, C., Gervain, J., Hamlin, J. K., Hannon, E. E., Kline, M., Levelt, C., Lew-Williams, C., Nazzi, T., Panneton, R., Rabagliati, H., Soderstrom, M., Sullivan, J., Waxman, S., \& Yurovsky, D. (2017). A Collaborative Approach to Infant Research: Promoting Reproducibility, Best Practices, and Theory-Building. Infancy, 22(4), 421-435. https://doi.org/10.1111/infa.12182

Gennetian, L. A., Tamis-LeMonda, C. S., \& Frank, M. C. (2020). Advancing Transparency and Openness in Child Development Research: Opportunities. Child development perspectives, 14(1), 3-8. https://doi.org/10.1111/cdep.12356

Gilmore, R. O., \& Qian, Y. (2021). An open developmental science will be more rigorous, robust, and impactful. Infant and Child Development, e2254.

https://doi.org/10.1002/icd.2254

Havron, N., Bergmann, C., \& Tsuji, S. (2020). Preregistration in infant research-A primer. Infancy, 25(5), 734-754. https://doi.org/10.1111/infa.12353

Higginson, A. D., \& Munafò, M. R. (2016). Current Incentives for Scientists Lead to Underpowered Studies with Erroneous Conclusions. PLoS biology, 14(11), e2000995. https://doi.org/10.1371/journal.pbio.2000995

Higginson, A. D., \& Munafò, M. R. (2016). Current Incentives for Scientists Lead to Underpowered Studies with Erroneous Conclusions. PLoS biology, 14(11), e2000995. https://doi.org/10.1371/journal.pbio.2000995

Hobson, H. (2019). Registered reports are an ally to early career researchers. Nature Human Behavior, 3, 1010. doi:10.1038/ s41562-019-0701-8 
OPEN DEVELOPMENTAL SCIENCE

Holcombe, A. O. (2019, April 18). Contributorship, Not Authorship: Use CRediT to Indicate Who Did What. https://doi.org/10.3390/publications7030048

John, L. K., Loewenstein, G., \& Prelec, D. (2012). Measuring the prevalence of questionable research practices with incentives for truth telling. Psychological Science, 23(5), 524-532. https://doi.org/10.1177/0956797611430953

Plucker, J. A., \& Makel, M. C. (2021). Replication is important for educational psychology: Recent developments and key issues. Educational Psychologist, 56(2), 90100. https://doi.org/10.1080/00461520.2021.1895796

Kathawalla, U. K., Silverstein, P., \& Syed, M. (2021). Easing into open science: A guide for graduate students and their advisors. Collabra: Psychology, 7(1), 18684. https://doi.org/10.1525/collabra.18684

Kathawalla, U.-K., Silverstein, P., \& Syed, M. (2021). Easing into open science: A guide for graduate students and their advisors. Collabra: Psychology, 7(1), 18684. doi:10.1525/collabra. 18684

Kessler, A. M., Likely, R., \& Rosenberg, J. (2021, February 4). Open for Whom? The Need to Define Open Science for Science Education. https://doi.org/10.31219/osf.io/sqcn7

Kim et al., (2020). Teaching and mentoring open science. https://osf.io/jux4t/

Kowalczyk, O., Lautarescu, A., Blok, E., Dall’Aglio, L., \& Westwood, S. J. (2020). What senior academics can do to support reproducible and open research: A short, three-step guide. PsyArXiv. doi:10.31234/osf.io/jyfr7

Logan, J. A. R., Hart, S. A., \& Schatschneider, C. (2021). Data Sharing in Education Science. AERA Open. https://doi.org/10.1177/23328584211006475 
OPEN DEVELOPMENTAL SCIENCE

Lundwall R. A. (2019). Changing institutional incentives to foster sound scientific practices: One department. Infant behavior \& development, 55, 69-76. https://doi.org/10.1016/j.infbeh.2019.03.006

Makel, M. C., Smith, K. N., McBee, M., Peters, S. J., \& Miller, E. M. (2019, March 26). Open Science 2.0: Large-Scale Collaborative Education Research. https://doi.org/10.31234/osf.io/ypmjg

Mannheimer, S., Pienta, A., Kirilova, D., Elman, C., \& Wutich, A. (2019). Qualitative Data Sharing: Data Repositories and Academic Libraries as Key Partners in Addressing Challenges. American Behavioral Scientist, 63(5), 643664. https://doi.org/10.1177/0002764218784991

Meehl, P. E. (1967). Theory-Testing in Psychology and Physics: A Methodological Paradox. Philosophy of Science, 34(2), 103-115. http://www.jstor.org/stable/186099

Meyer, M. N. (2018). Practical Tips for Ethical Data Sharing. Advances in Methods and Practices in Psychological Science, 131-144. https://doi.org/10.1177/2515245917747656

Munafò, M. R., Nosek, B. A., Bishop, D. V., Button, K. S., Chambers, C. D., Du Sert, N. P., ... \& Ioannidis, J. P. (2017). A manifesto for reproducible science. Nature human behaviour, 1(1), 1-9. https://doi.org/10.1038/s41562-016-0021

Norris, E., \& O'Connor, D. B. (2019). Science as behaviour: Using a behaviour change approach to increase uptake of open science. Psychology \& Health, 34(12), 1397 1406. doi:10.1080/ 08870446.2019.1679373 
OPEN DEVELOPMENTAL SCIENCE

Nosek, B. A., Alter, G., Banks, G. C., Borsboom, D., Bowman, S. D., Breckler, S. J., ... \& Yarkoni, T. S. (2015). Promoting an open research culture. Science, 348(6242), $1422-$ 1425. https://doi.org/10.1126/science.aab2374

Nosek, B. A., Ebersole, C. R., DeHaven, A. C., \& Mellor, D. T. (2018). The preregistration revolution. Proceedings of the National Academy of Sciences of the United States of America, 115(11), 2600-2606. https://doi.org/10.1073/pnas.1708274114

Parsons, S., Azevedo, F., Elsherif, M. M., Guay, S., Shahim, O. N., Govaart, G. H., Norris, E., O’Mahony, A., Parker, A. J., Todorovic, A., Pennington, C. R., GarciaPelegrin, E., Lazić, A., Robertson, O. M., Middleton, S. L., Valentini, B., McCuaig, J., Baker, B. J., Collins, E., ... Aczel, B. (2021). A Community-Sourced Glossary of Open Scholarship Terms [Manuscript submitted for publication]. Department of Experimental Psychology, University of Oxford

Pashler, H., \& Wagenmakers, E. (2012). Editors' Introduction to the Special Section on Replicability in Psychological Science: A Crisis of Confidence? Perspectives on Psychological Science, 7(6), 528-530. https://doi.org/10.1177/1745691612465253

Peterson, D. (2016). The Baby Factory: Difficult Research Objects, Disciplinary Standards, and the Production of Statistical Significance. Socius. https://doi.org/10.1177/2378023115625071

Pownall, M., Azevedo, F., Aldoh, A., Elsherif, M. M., Vasilev, M. R., Pennington, C. R., ... Parsons, S. (2021, July 28). Embedding open and reproducible science into teaching: A bank of lesson plans and resources. https://doi.org/10.31234/osf.io/fgv79 
OPEN DEVELOPMENTAL SCIENCE

Reich, J., Gehlbach, H., \& Albers, C. J. (2020). "Like upgrading from a typewriter to a computer": Registered reports in education research. AERA Open, 6(2), 1-6. https://doi.org/10.1177/2332858420917640

Roehrig, A. D., Soper, D., Cox, B. E., \& Colvin, G. P. (2018). Changing the Default to Support Open Access to Education Research. Educational Researcher, 47(7), 465473. https://doi.org/10.3102/0013189X18782974

Simmons, J. P., Nelson, L. D., \& Simonsohn, U. (2011). False-Positive Psychology: Undisclosed Flexibility in Data Collection and Analysis Allows Presenting Anything as Significant. Psychological Science, 22(11), 1359-

1366. https://doi.org/10.1177/0956797611417632

Silverstein, Priya (2020) Evaluating the replicability and specificity of evidence for natural pedagogy theory. $\mathrm{PhD}$ thesis.

https://eprints.lancs.ac.uk/id/eprint/148689/1/2020silversteinphd.pdf

SRCD. (2019). Policy on Scientific Integrity, Transparency, and Openness j Society for Research in Child Development SRCD. Retrieved from https://www.srcd.org/policyscientific-integrity-transparency-and-openness

Stojmenovska, D., Bol, T., \& Leopold, T. (2019). Teaching Replication to Graduate Students. Teaching Sociology, 47(4), 303-313. https://doi.org/10.1177/0092055X19867996

Strand, J. F., \& Brown, V. A. (2019). Publishing Open, Reproducible Research With Undergraduates. Frontiers in psychology, 10, 564. https://doi.org/10.3389/fpsyg.2019.00564 
OPEN DEVELOPMENTAL SCIENCE

Syed, M. (2019, April 15). The Open Science Movement is For All of Us. https://doi.org/10.31234/osf.io/cteyb

Syed, M., \& Kathawalla, U. K. (2022). Cultural psychology, diversity, and representation in open science. In K. C. McLean (Ed.), Cultural methods in psychology: Describing and transforming cultures (pp. 427-454). New York: Oxford University Press

Terry, N.P, Petscher, Y., Gaab, N., \& Hart, S.A. (2021). Researchers translating the science of reading: Widening the lens of translational science through team science. The Reading League Journal, 2(1), 46-53.

Travers, J. C., Cook, B. G., Therrien, W. J., \& Coyne, M. D. (2016). Replication Research and Special Education. Remedial and Special Education, 37(4), 195-204. https://doi.org/10.1177/0741932516648462

Tsai, A. C., Kohrt, B. A., Matthews, L. T., Betancourt, T. S., Lee, J. K., Papachristos, A. V., Weiser, S. D., \& Dworkin, S. L. (2016). Promises and pitfalls of data sharing in qualitative research. Social Science \& Medicine, 169, 191-198. doi:10.1016/j. socscimed.2016.08.004

*van Dijk, W., Schatschneider, C., \& Hart, S. A. (2021). Open Science in Education Sciences. Journal of Learning Disabilities, 54(2), 139-152. https://doi.org/10.1177/0022219420945267

Washburn, A. N., Hanson, B. E., Motyl, M., Skitka, L. J., Yantis, C., Wong, K. M., ... Carsel, T. S. (2018). Why do some psychology researchers resist adopting proposed reforms to research practices? A description of researchers' rationales. Advances in Methods and Practices in Psychological Science, 1, 166-173. https://doi.org/10.1177/2515245918757427 
OPEN DEVELOPMENTAL SCIENCE

Whitaker, T., \& Guest, O. (2020). \#bropenscience is broken science. The Psychologist, 33, 34-38. https://thepsychologist. bps.org.uk/volume-33/november2020/bropenscience-brokenscience

Wicherts, J. M., Veldkamp, C. L., Augusteijn, H. E., Bakker, M., van Aert, R. C., \& van Assen, M. A. (2016). Degrees of Freedom in Planning, Running, Analyzing, and Reporting Psychological Studies: A Checklist to Avoid p-Hacking. Frontiers in psychology, 7, 1832. https://doi.org/10.3389/fpsyg.2016.01832

Wilkinson, M. D., Dumontier, M., Aalbersberg, I. J., Appleton, G., Axton, M., Baak, A., ... \& Mons, B. (2016). The FAIR Guiding Principles for scientific data management and stewardship. Scientific data, 3(1), 1-9. https://doi.org/10.1038/sdata.2016.18 